\title{
Evidencia proveniente de estudios en modelos animales acerca de los efectos del ejercicio y el enriquecimiento ambiental sobre la neurogénesis en el adulto
}

\author{
Bryan Montero-Herrera \\ Centro de Investigación en Neurociencias, Universidad de Costa Rica, Costa Rica
}

\begin{abstract}
Resumen
Referirse a neurogénesis hace parecer que sea un tema muy reciente en el campo de la ciencia, pero la realidad es que se está investigando aproximadamente desde 1962, año en el que apareció el primer experimento que usaba modelos animales para medir el incremento en la cantidad de neuronas en ciertas zonas del cerebro. Desde ese momento se han desarrollado muchas investigaciones que buscan descubrir cómo se lleva a cabo este proceso y qué factores lo modulan. Esta revisión se enfoca en el ejercicio (aeróbico y/o contra resistencia) y el enriquecimiento ambiental (EA) como dos de las condiciones principales que se han asociado con la neurogénesis en el adulto. Los resultados de los artículos demuestran que tanto el ejercicio como el EA promueven la formación de nuevas neuronas, aunque el ejercicio produce mayores efectos.
\end{abstract}

Palabras clave: Ejercicio aeróbico. Ejercicio contra resistencia. Ambiente enriquecido. Neurogénesis. Factor neurotrófico derivado del cerebro (BDNF).

\section{Evidence coming from animal model studies about exercise effects and environmental enrichment on adults neurogenesis}

\begin{abstract}
Referring to neurogenesis seems to be a very recent issue in the field of science, but the reality is that it has been investigated since the year 1962, when appeared the first experiment using animal models to measure the increase of neurons amount in certain areas of the brain. Since that time, many researches have been developed that seek to discover how this process is carried out and what factors modulate it. This review focuses on exercise and environmental enrichment as two of the main associated conditions that have been associated with neurogenesis. This review focuses on exercise (aerobic and/or resistance) and environmental enrichment as two of the main conditions that have been associated with neurogenesis in adults. The results of the articles show that both exercise and environmental enrichment promote the formation of new neurons, however, exercise produces greater effects.
\end{abstract}

Key words: Aerobic exercise. Resistance training. Enriched environment. Neurogenesis. Brain derived neurotrophic factor (BDNF).

Correspondencia:

Bryan Montero-Herrera

E-mails: bryan_mh2005@ hotmail.com
Fecha de recepción: 18-10-2017

Fecha de aceptación: 12-10-2018

DOI: 10.24875/RMN.M18000016
Disponible en internet: 03-01-2019 Rev Mex Neuroci. 2018;19(6):53-69 www.revmexneurociencia.com 1665-5044/@ 2018. Academia Mexicana de Neurología A.C. Publicado por Permanyer México. Este es un artículo Open Access bajo la licencia CC BY-NC-ND (http://creativecommons.org/licenses/by-nc-nd/4.0/). 


\section{Introducción}

Hasta la fecha se han realizado numerosas investigaciones que evidencian los beneficios de la práctica de ejercicio (EJF) o actividad física regular en la salud física, fisiológica y psicológica-mental de las personas ${ }^{1-5}$. Estos estudios se han desarrollado con poblaciones que padecen o han padecido alguna enfermedad, así como también en personas sanas, y en ambos casos los resultados obtenidos han sido positivos. Por mencionar algunos: mejora la resistencia, la fuerza, el autoconcepto físico, la motivación y la presión arterial, entre otros ${ }^{1,2}$.

Debido a todos los cambios que se producen en el cuerpo durante y después de la realización del EJF, los investigadores consideran al EJF como la «polipíldora", es decir, un proceso que se encarga de liberar una gran cantidad de sustancias, como interleucina (IL) 6 (IL-6), IL-3, IL-4, factor neurotrófico derivado del cerebro (BDNF), factor de crecimiento insulínico 1 (IGF-1) y factor de crecimiento vascular endotelial (VEGF), entre otras, que se activan por medio del EJF y que van a actuar en diferentes zonas del organismo y a provocar una cascada de reacciones en diversos sistemas y órganos ${ }^{6}$.

De las proteínas mencionadas anteriormente, se van a considerar principalmente dos, que actúan a nivel del cerebro favoreciendo el proceso conocido como neurogénesis. Éstas son el BDNF y el IGF-1.

En esta revisión se define neurogénesis como la formación de nuevas neuronas en el sistema nervioso central a partir de una célula madre ${ }^{7}$. Estas nuevas neuronas cuentan con más ramificaciones dendríticas y cierta densidad de las espinas dendríticas, lo que aumenta la probabilidad de que ocurran sinapsis (proceso por el cual las neuronas se comunican unas con otras) entre ellas y las neuronas ya formadas. Se distinguen dos tipos de neurogénesis: una que se lleva a cabo en la etapa prenatal y posnatal (antes de ingresar en la etapa adulta), la cual se conoce desde hace mucho tiempo, y otra que ocurre en la etapa adulta. El descubrimiento de este segundo tipo rompió un paradigma muy antiguo, pues antes se pensaba que en el adulto no podían formarse ni desarrollarse nuevas células nerviosas ${ }^{8,9}$. El presente artículo se centrará en este tipo de neurogénesis.

Existen diferentes condiciones que se han asociado con un incremento en la neurogénesis en el adulto, entre las que destacan el EJF y el EA. Este último se refiere a una condición en la que se estimulan los sentidos de los sujetos (sean animales o personas) incluyendo en un ambiente particular componentes de interacción social (varios individuos en un mismo lugar) y/o componentes sensoriales de diferentes modalidades (visuales, táctiles, olfatorios o propioceptivos) ${ }^{10-12}$. Otra particularidad de los EA es que los elementos sensoriales se van cambiando constantemente, con el objetivo de estimular los diferentes sentidos de los participantes (personas o animales) para que no se habitúen a una sola condición ${ }^{13,14}$.

\section{Definición y formación de la neurogénesis}

A día de hoy se sabe que la neurogénesis se presenta en las diferentes etapas del desarrollo del ser humano (prenatal, nacimiento, niñez, adolescencia, adultos y adultos mayores); no obstante, algunos autores aclaran que los investigadores tenían la idea de que la neurogénesis se iniciaba en la etapa prenatal y continuaba aproximadamente hasta la adolescencia ${ }^{8,9}$. Pero con la aparición de diversos estudios se logró aportar evidencia para probar que la neurogénesis también se desarrolla en personas adultas e incluso en personas adultas mayores ${ }^{15,16}$.

Es importante distinguir los términos de neurogénesis y neuroplasticidad, ya que en ocasiones son considerados como dos procesos similares, pero en realidad no lo son. Por un lado, la neurogénesis en el adulto es la formación de nuevas neuronas en esa etapa del desarrollo y se da específicamente en dos zonas del cerebro: el giro dentado y el bulbo olfatorio ${ }^{17}$. Por el otro lado, la neuroplasticidad es la capacidad del cerebro para modificar sus conexiones sinápticas como respuesta a los cambios a los que se ve sometido el individuo ${ }^{18}$. Algunas de las modificaciones son: aumento en el número de sinapsis y células gliales, e incremento en las prolongaciones de las espinas dendríticas ${ }^{19}$.

En el adulto, la formación de nuevas neuronas puede darse en dos zonas del cerebro (bulbo olfatorio y giro dentado del hipocampo) en las que ocurren la división celular, la migración y la diferenciación ${ }^{20,21}$. La neurogénesis comienza con la activación de las células madre; si éstas se alojan en el bulbo olfatorio, la neurogénesis se produce en la zona subventricular (ZSV), y si corresponden a las del giro dentado, se produce en la zona subgranular (ZSG) ${ }^{22}$.

Las células madre en su proceso de división pueden convertirse en neuronas 0 en neuroglía. Estas células migran (Fig. 1) hacia la ZSV o ZSG y de ahí se desplazan hacia el giro dentado o el bulbo olfatorio en una fase conocida como "cadena rostral migratoria» ${ }^{23}$. Una vez alojadas en las zonas correspondientes, las células que 


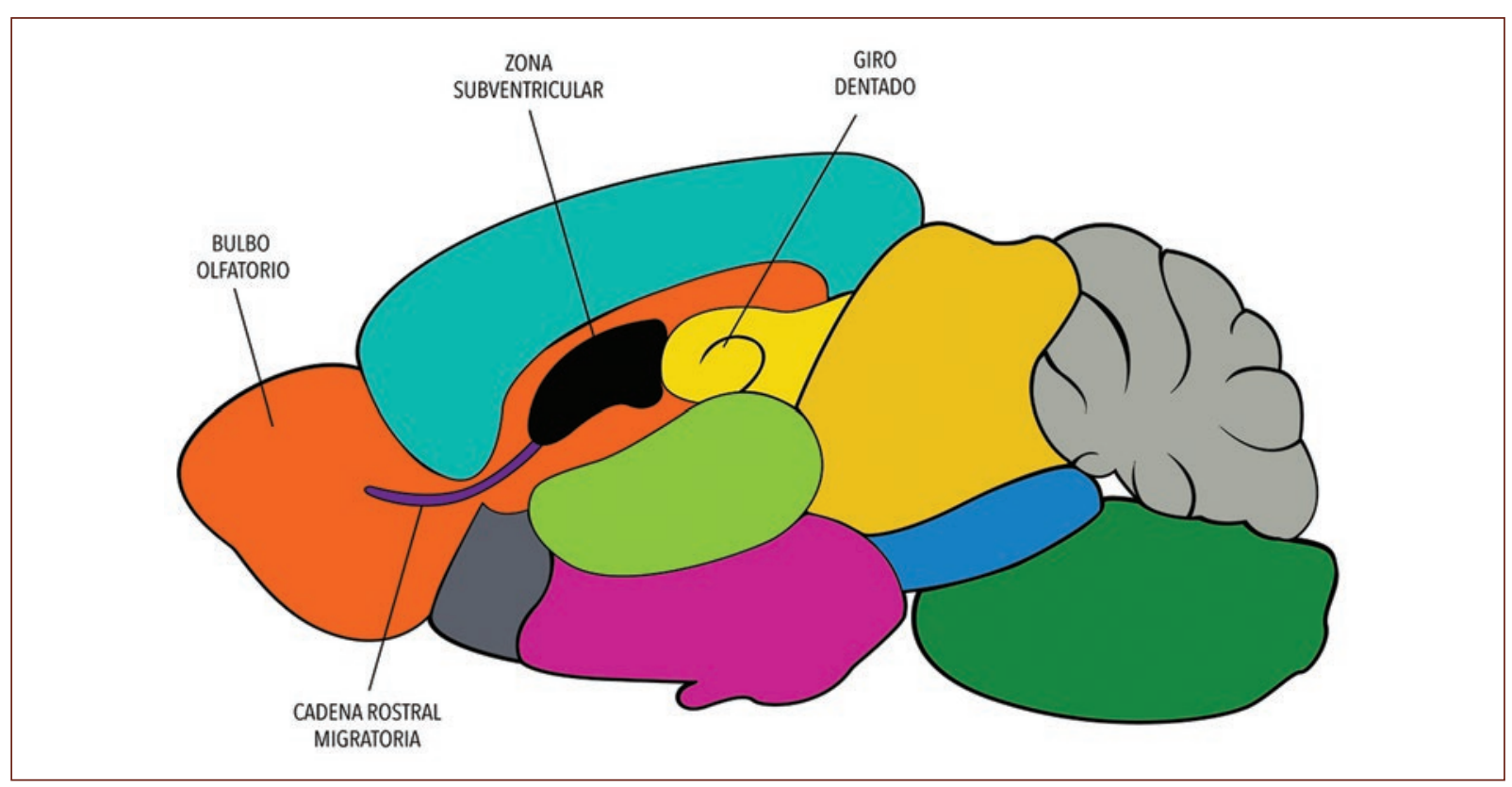

Figura 1. Zonas cerebrales en las que ocurre el proceso de neurogénesis presentes en ratas. Vista sagital de un cerebro de rata adulta donde se detallan las dos regiones en las cuales se conoce que la neurogénesis se lleva a cabo en la etapa adulta: el giro dentado en la formación hipocampal y la ZSV del bulbo olfatorio (imagen original de Montero Herrera $B$ y Cambronero $E$, 2017).

lograron formarse como neuronas (etapa de diferenciación) tardarán entre cuatro y siete semanas para establecer sinapsis con las neuronas que ya se encontraban en esa región cerebral (etapa de sinaptogénesis), completando con esto el proceso de neurogénesis ${ }^{23}$.

\section{Historia de la neurogénesis}

La neurogénesis es uno de esos casos en los cuales se rompió un mito en la sociedad y un paradigma de las neurociencias, pues se pensaba que el nacimiento de neuronas en el cerebro del adulto era algo imposible. Los primeros estudios elaborados en el área de la neurogénesis se atribuyen a Altman, quien en 1962 y posteriormente en 1965 (junto con G. Das) logró probar por vez primera el surgimiento de nuevas neuronas en el bulbo olfatorio y el giro dentado del hipocampo de ratas adultas 24,25 .

El primer artículo en sugerir que este proceso también se presentaba en el cerebro de las personas adultas fue llevado a cabo por Eriksson, et al. en 1998 en pacientes con cáncer ${ }^{16}$ que recibieron inyecciones de bromodesoxiuridina (BrdU). Este método permite estudiar la fase en la cual las células se están replicando y donde nuevas copias de ADN están siendo formadas ${ }^{26}$. Los autores observaron que con estas inyecciones había nuevas neuronas en la zona del giro dentado. Cabe resaltar que el objetivo inicial con la BrdU era cuantificar las células tumorales, pero terminaron descubriendo la neurogénesis en el adulto ${ }^{16}$.

Tras este descubrimiento, algunos investigadores plantearon la hipótesis de si habría alguna modificación en la cantidad de neuronas producidas dependiendo de la actividad que hicieran las ratas ${ }^{27}$. Así pues, colocaron a animales en cuatro condiciones diferentes (nadar, correr, EA y control) con el fin de estudiar los posibles cambios en el cerebro. $Y$ demostraron que el número de neuronas producidas al correr o con EA es mayor $(p<0.05)$ que nadando; por su parte, la condición control no mostró cambios notorios. Este estudio fue pionero y motivó nuevas líneas de investigación en materia de la neurogénesis, principalmente relacionadas con el EJF y el EA ${ }^{28-30}$.

\section{Principales factores promotores: ejercicio y enriquecimiento ambiental}

Como se mencionó previamente, el EJF y el EA generan cambios a nivel cerebral que favorecen la neurogénesis. A continuación, se exponen las dos condiciones (EJF y EA) por separado para analizar los efectos que conllevan cada una de ellas y luego se comentarán artículos en los 
que se combinan ambas condiciones. Surge, por lo tanto, la pregunta de si bajo esas condiciones se producirá más o menos neurogénesis.

\section{Ejercicio}

En diferentes estudios ${ }^{30,31}$ se ha demostrado que el EJF tiene una activación cortical y cerebral más alta en comparación con el EA, lo cual se traduce en un incremento del volumen sanguíneo y el flujo cerebral ${ }^{32}$, así como también en un incremento en la angiogénesis y la sinaptogénesis ${ }^{33}$, un mayor aporte de glucosa ${ }^{34} \mathrm{y}$ un aumento de proteínas como BDNF e IGF-1 en sangre ${ }^{35-37}$.

En 2011, un grupo de científicos sometieron unas ratas Wistar de dos meses a diferentes condiciones y establecieron cuatro grupos: el primero fue el de ratas sedentarias que no se ejercitaron y los otros tres grupos se ejercitaron en una banda sin fin bajo diferentes programas, pero todos durante $40 \mathrm{~min} /$ día a $10 \mathrm{millas} / \mathrm{h}$ $(0.6 \mathrm{~km} / \mathrm{h}$ ). El segundo grupo (el primero de las ratas ejercitadas) siguió el programa durante tres días, el tercero por siete días y el último durante 15 días $^{38}$.

Al finalizar, todos los grupos de animales ejercitados presentaron niveles mayores de BDNF en el hipocampo, en comparación con los animales sedentarios. Entre los grupos ejercitados, el que trabajó por 15 días mostró los niveles más altos para este factor, aunque también hay estudios en los que se alcanzaron incrementos en el BDNF en ratas que corrieron por tres y siete días ${ }^{28}$.

En otro estudio se aplicaron tres experimentos al mismo tiempo con el fin de medir la neurogénesis como respuesta a diferentes condiciones: el primer experimento consistía en comparar un grupo de ratas sedentarias, otras que corrían cada cierto tiempo, un tercer grupo que tenía a disposición la banda para correr cuando quisieran (lo hacían voluntariamente) y las últimas pertenecían al grupo HIIT (High intensity interval training), las cuales realizaban $5 \mathrm{~min}$ de EJF al $50-60 \%$ y 3 min al $85-90 \%$; para mantener dicha intensidad tenían un sistema (rejilla electrificada) para que en caso de que la bajaran volvieran a aumentarla. En total estuvieron en estudio durante siete semanas ${ }^{29}$.

Los autores encontraron que la neurogénesis fue mayor en los grupos que practicaron EJF aeróbico (siendo más alta en aquellas ratas que corrieron más distancia en la banda), en comparación con todos los demás grupos: los sedentarios, los HIIT y los que llevaron a cabo EJF contra resistencia ${ }^{29}$. Estos datos concuerdan con los de otros autores, que también encuentran efectos a favor del EJF aeróbico ${ }^{39,40}$.
Respecto al EJF contra resistencia, algunos investigaciones han hallado incrementos de la proteína IGF-1 tanto en sangre como a nivel del hipocampo (la cual es una de las promotoras de la neurogénesis). En este estudio las ratas trabajaron siete semanas, un total de cinco veces por semana, de ahí que los resultados fueran diferentes ${ }^{39,41}$. Cabe destacar que el EJF aeróbico incrementa más el BDNF y el EJF contra resistencia el IGF-142-44.

\section{Enriquecimiento ambiental}

La mayoría de las investigaciones sobre EA se han realizado exponiendo a los animales a dos tipos de enriquecimiento: el primero es de tipo físico, en el que se incluyen elementos como juguetes, túneles o ruedas para hacer EJF en cada una de las jaulas, y el segundo es enriquecimiento social, en el que se favorece la interacción entre animales alojándolos juntos en una jaula ${ }^{45,46}$.

Es normal ver dentro de los estudios de EA la colocación de ruedas para hacer EJF; no obstante, ya anteriormente se comentó acerca de los beneficios del EJF en los procesos de la neurogénesis, de ahí que en este apartado sea importante exponer trabajos en los que la variable EJF no esté presente; sin embargo, en caso de que hubiera EJF, éste debe realizarse en una caja aparte de las otras condiciones de EA (sensorial o social), con el fin de posibilitar tener un mejor panorama sobre los beneficios que brinda el EA por sí sólo, y no combinándolo con el EJF.

Para llevar a cabo esta investigación se utilizaron dos grupos de ratones: un grupo fue mantenido en condiciones estándar $(26 \times 42 \times 18 \mathrm{~cm})$ y en el otro se mantenían de cinco a seis ratones en una caja grande $(44 \times 62 \times$ $28 \mathrm{~cm}$ ) con tubos de comida, rueda, túneles, escaleras y refugios. Además, cada uno de estos elementos se cambiaba de posición diariamente y se sustituían por otros cada semana. El estudio mostró un aumento de aproximadamente el doble en el número de neuronas (medido con BrdU) y un incremento en los niveles de BDNF en el giro dentado en el grupo de cinco a seis ratones en la caja grande, con respecto a los animales que fueron alojados en condiciones estándar ${ }^{47}$.

En otro artículo, pero utilizando únicamente ratones hembra de tres meses de edad, los investigadores formaron tres grupos: el primero era de control, el segundo realizó EJF en una rueda y el tercero estaba en un EA (túneles, juguetes y materiales de nido). A los 13 días de haber comenzado el experimento, seis ratones de cada condición fueron escogidos para 
estudiar los cambios neuronales (usando BrdU) en el bulbo olfatorio y el hipocampo. Posteriormente, los resultados fueron comparados con los de otros ocho ratones que permanecieron en sus cajas por 43 días más (ya que éste es un tiempo suficiente para que las nuevas células migren hacia una determinada zona y logren diferenciarse), y así poder establecer cuántas neuronas nuevas se habían formado en ese tiempo ${ }^{48}$.

En las conclusiones del experimento anterior se hace énfasis en que situaciones de EA también son beneficiosas para el incremento en el número de neuronas. Estos cambios suceden tanto en el bulbo olfatorio como en el hipocampo, siendo el hipocampo el área cerebral que más neurogénesis presenta cuando se compara con el bulbo olfatorio ${ }^{48}$.

Más recientemente, en otro estudio se agruparon ratones Swiss macho con edades comprendidas entre 25 y 30 días u 8-12 semanas en cuatro condiciones diferentes: cinco ratones en una caja con condición estándar, un único ratón en una caja estándar, cinco ratones en una caja con EA (refugio de plástico, dos rollos de cartón, tres juguetes y cintas con texturas distintas) y el último grupo era un único ratón en un EA igual al anterior. Los resultados muestran que las condiciones en las que se presentaban algún tipo de EA los valores de neurogénesis en el giro dentado y el bulbo olfatorio eran mayores. De igual forma, cuando se analizan los dos grupos sin ningún tipo de EA, se observa que existe formación de nuevas neuronas en la caja que contenía varios ratones, mientras que en la del ratón solo esto no sucedió ${ }^{46}$.

Este estudio subraya la también importancia de la interacción social para favorecer el proceso de neurogénesis, pero resulta preciso resaltar que los resultados alcanzados en el número de neuronas que nacen son mayores cuando se combina la interacción social con un EA, que si solamente se presenta el factor social como variable. Aunadas a las comentadas en este apartado hay otras investigaciones que llegan a conclusiones similares ${ }^{49,50}$.

\section{Ejercicio y enriquecimiento ambiental}

Con el objetivo de estudiar los efectos del EJF y el EA independientemente e interactuando entre ellos, se realizó un estudio en dos partes. Para la primera se utilizaron un total de 40 ratones hembra distribuidas en cuatro grupos: control, EJF (dentro de una misma jaula había 10 ruedas para que corrieran, una para cada ratón), EA (túneles, iglúes, bolas, trozos de madera y cabañas para escalar) y EA más EJF (se incluían los elementos que se mencionaron anteriormente para la condición de EA y EJF). En los primeros 12 días los animales recibieron inyecciones de BrdU, y el día 13 se tomaron cinco ratones de cada grupo para analizar los cambios a nivel neuronal; los restantes permanecieron hasta el final del estudio (día 43) (1). $^{\text {. }}$

En el caso del segundo experimento se utilizó una muestra de 48 ratones hembra divididas en los mismos grupos utilizados en el primer experimento (control, EJF, EA y EA con EJF) y además se aplicó una prueba de campo abierto (el día 30) durante 20 min con el objetivo de medir su desplazamiento. Este experimento se prolongó también durante 43 días.

Los resultados en ambos experimentos demostraron que los niveles de BDNF y la cantidad de neuronas que se obtienen en las condiciones en las que se realiza EJF corriendo en la rueda son significativamente más elevados que en la condición control y en la condición en la que los animales eran expuestos únicamente al EA. A partir de los datos obtenidos, los autores sugieren que tanto el EJF como el EA permiten el incremento en el número de neuronas ${ }^{51-53}$.

En un experimento similar al anterior se analizan 32 ratones en diferentes situaciones: ocho ratones en una condición control, ocho ratones en un EA (lecho, juguetes, bebidas dulces y golosinas de comidas), ocho ratones que hacían EJF voluntariamente en una rueda y los últimos ocho tenían una combinación de EA con ruedas para correr. En cada uno de estos ambientes los ratones estuvieron 32 días, en los cuales los primeros 10 días les administraron inyecciones con BrdU para identificar los cambios en el número de neuronas nuevas en el hipocampo ${ }^{54}$.

Se observó un incremento significativo en el número de neuronas en los animales que se encontraban en las dos condiciones que involucraban correr (EJF voluntario en una rueda y la combinación de EA con ruedas). La cantidad de nuevas neuronas que nacen por el EJF es mayor en términos de porcentaje en comparación con lo que aportan los EA: en el caso del EJF es de aproximadamente un $20 \%$ y en el EA de un $12 \%$. Otros autores señalan que tanto el EJF como el EA se complementan de varias formas por la activación de diferentes proteínas, como BDNF, IGF-1, VEGF, factor neurotrófico derivado de la glía) y factor de crecimiento neural (NGF), entre otras ${ }^{55-57}$.

Al inicio de este apartado se planteaba la pregunta de si al combinar el EJF con ambientes enriquecidos la neurogénesis sería mayor o menor. Con base en los aspectos comentados previamente, se puede notar que ambos métodos favorecen un aumento en la cantidad 
de células que se dividen a partir de las células ya existentes, aunque el EJF lo hace en mayor proporción que el EA.

Algunos investigadores ${ }^{58}$ proponen que el EA permite mantener con vida las nuevas células neuronales que nacen gracias a sus procesos de plasticidad sináptica, que incluye mejora de la arborización neuronal e incremento en el número de dendritas, mientras que el EJF se encarga de los procesos de proliferación celular, sobrevivencia celular y neurogénesis ${ }^{59,60}$. Para ver con más detalle algunos de los artículos que se mencionan dentro de este estudio, se puede consultar la tabla 1, en la cual se incluye información sobre autores, población, tipo de estudio, variable evaluada, tratamiento y conclusiones.

Aunque el número de trabajos realizados en seres humanos es reducido, los citados en este trabajo rescatan ciertos datos de interés ${ }^{41-44}$, por lo que resulta necesario analizar con cautela los resultados que se han ido obteniendo. Por ejemplo, aunque el EJF es la única variable que logró encontrarse aplicada en estudios llevados a cabo en personas, los efectos que se obtienen por medio de éste indican que hay cambios a nivel de proteínas importantes, como el BDNF y el IGF-1, que como se ha visto a lo largo del trabajo tienen un papel de suma importancia en la creación de nuevas neuronas en el cerebro adulto. Las mediciones de estas proteínas son a nivel periférico, pero se considera que si alguna de éstas se encuentra aumentada en esta parte del organismo, también lo estará a nivel central (cerebro), aunque la única forma de identificar la formación de nuevas células a nivel cerebral es aplicando estudios con metodologías que incluyan resonancia magnética funcional o tomografía por emisión de positrones.

Pero ¿qué ha pasado con la variable del EA? No hemos podido encontrar investigaciones que evaluaran los efectos del EA en personas, pues éste sólo ha sido analizado en modelos animales, y es a partir de ellos que sus efectos se han hecho notorios; no obstante, cabe mencionar que, si en modelos animales hay cambios que en ocasiones resultan ser significativos, sería importante ver qué sucede con las personas. Por ejemplo, si entrenar con más gente al mismo tiempo (interacción social) causa más liberación de proteínas que entrenar solo, o si los cambios constantes de materiales entre sesiones (interacción sensorial) aporta resultados más productivos en entrenamientos (liberación de mayor cantidad de proteínas) que los que generalmente se usan. Para estos ejemplos mencionados se

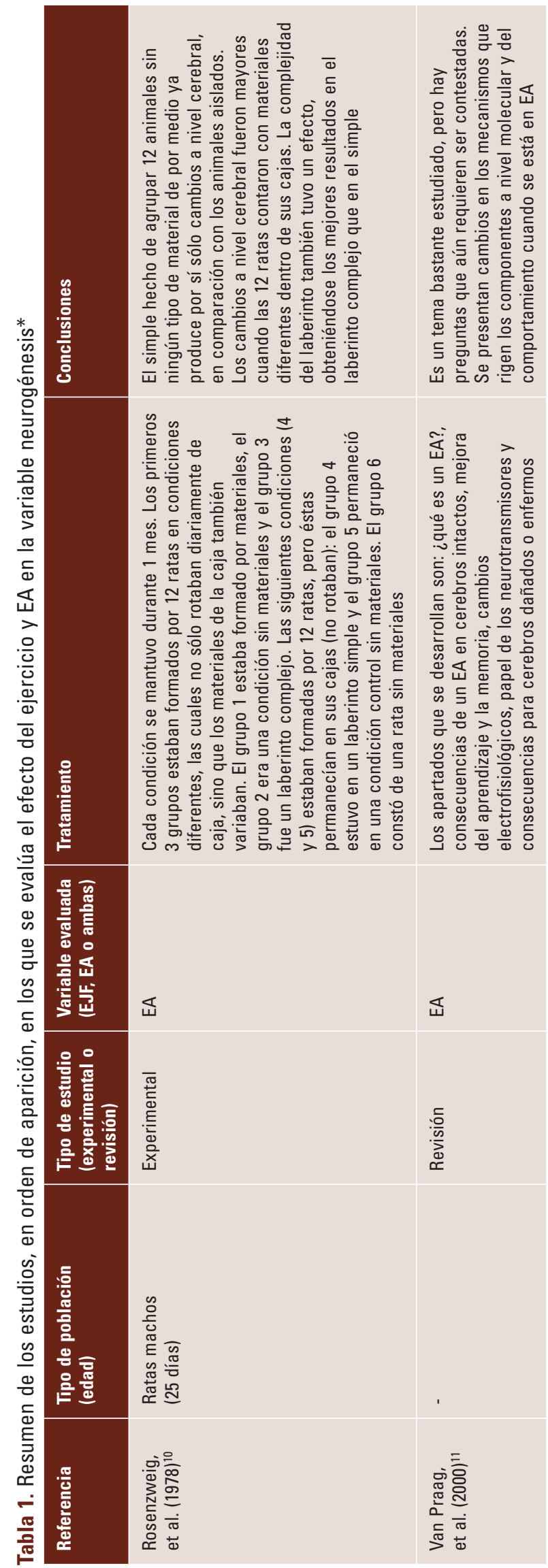




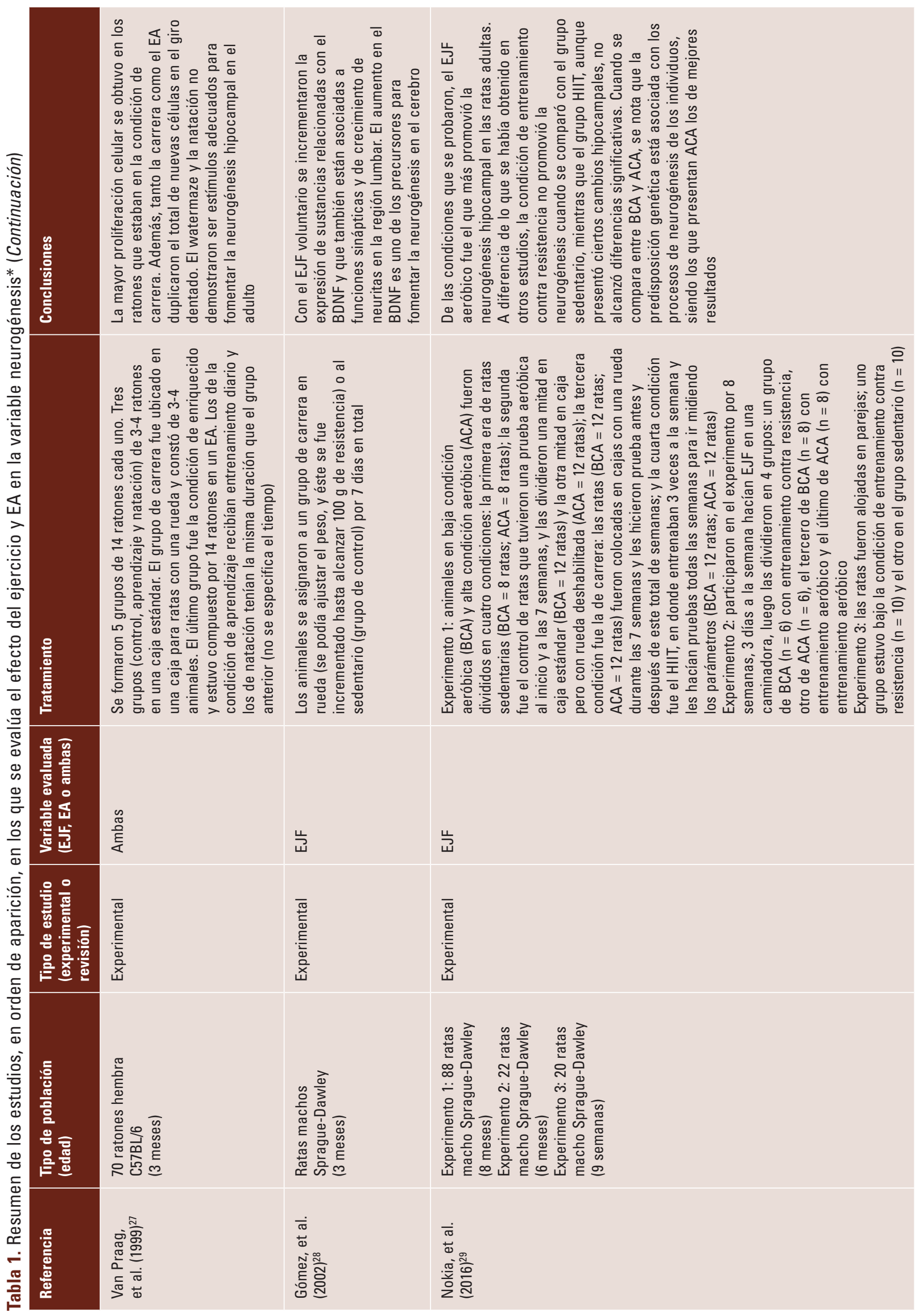



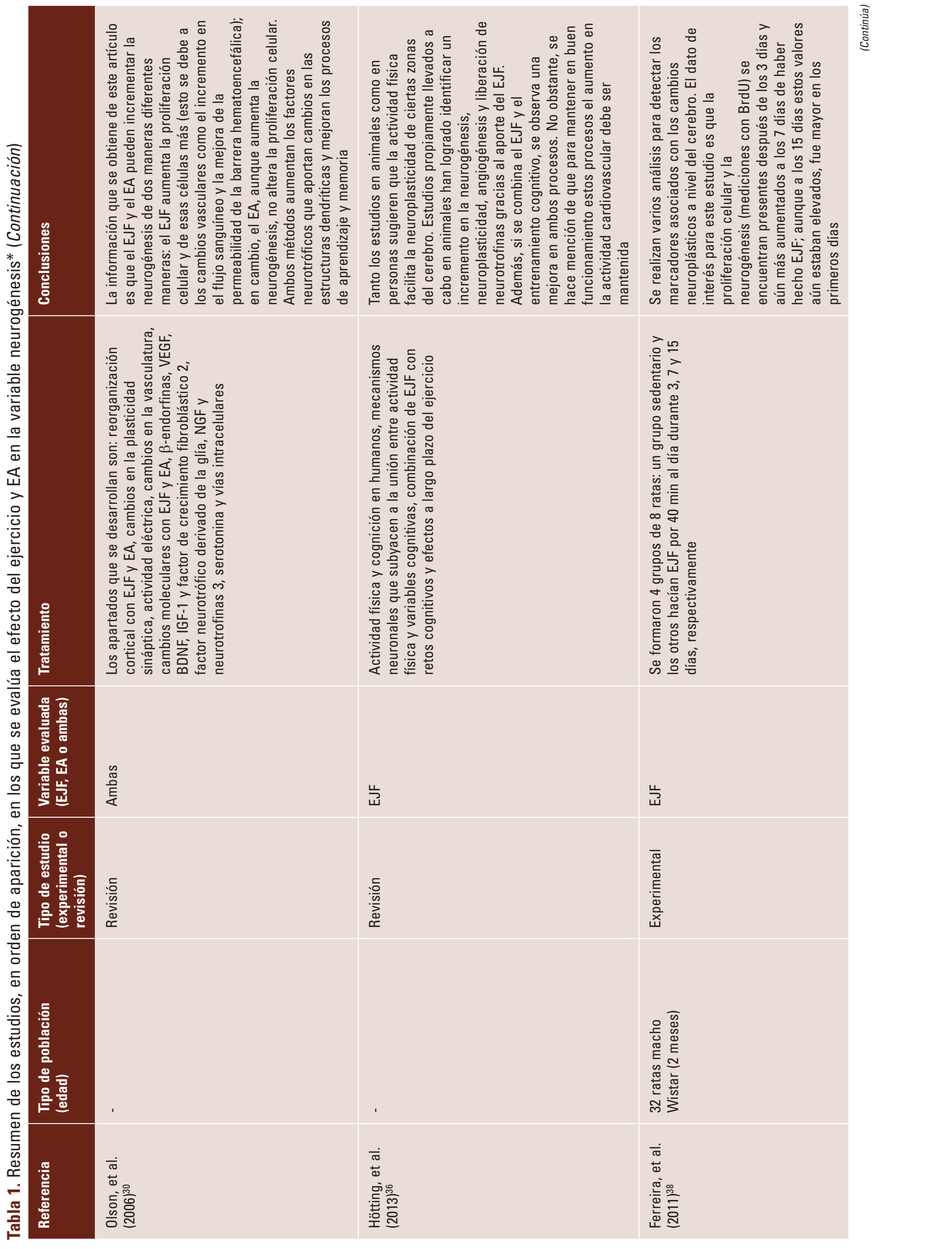


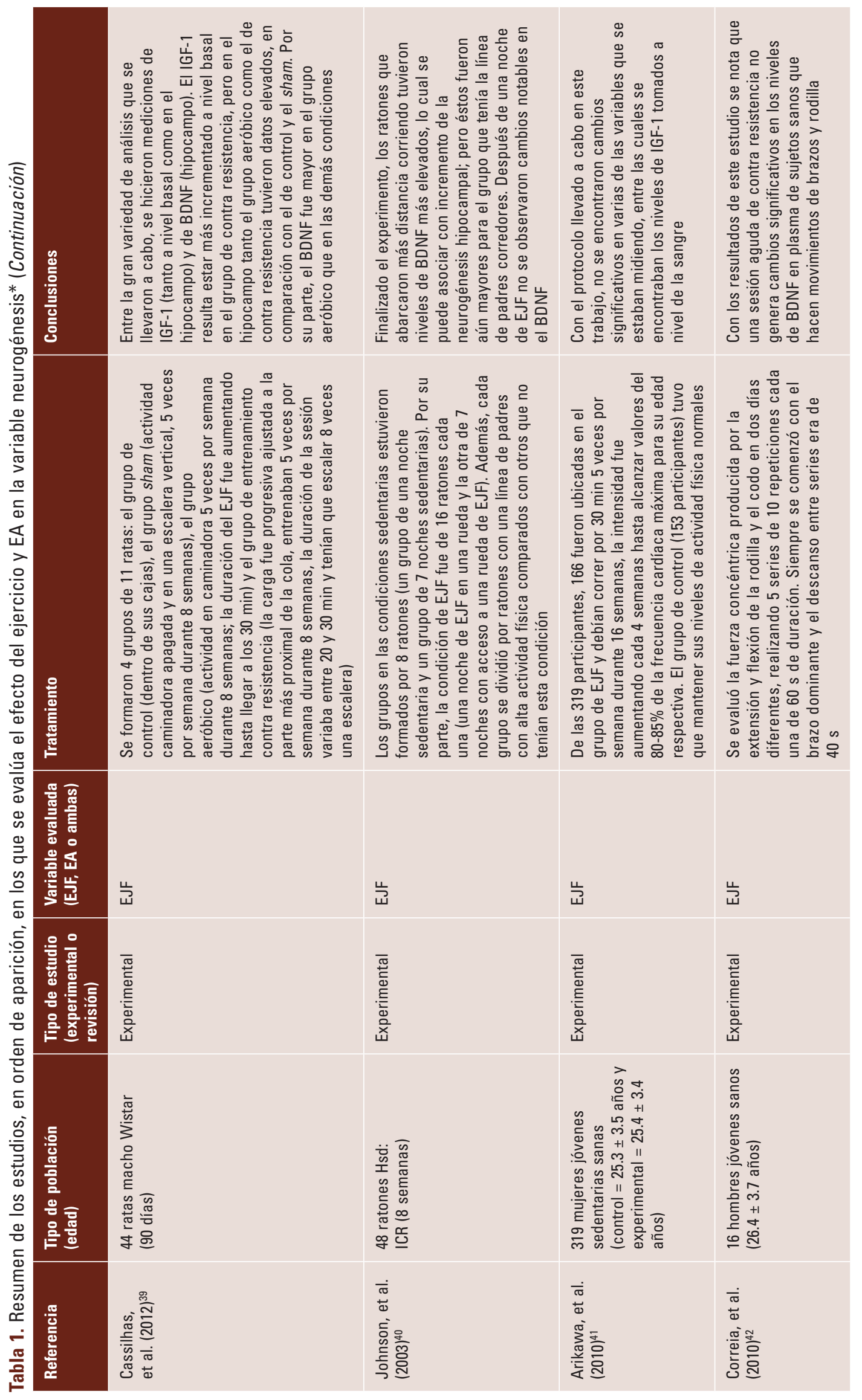




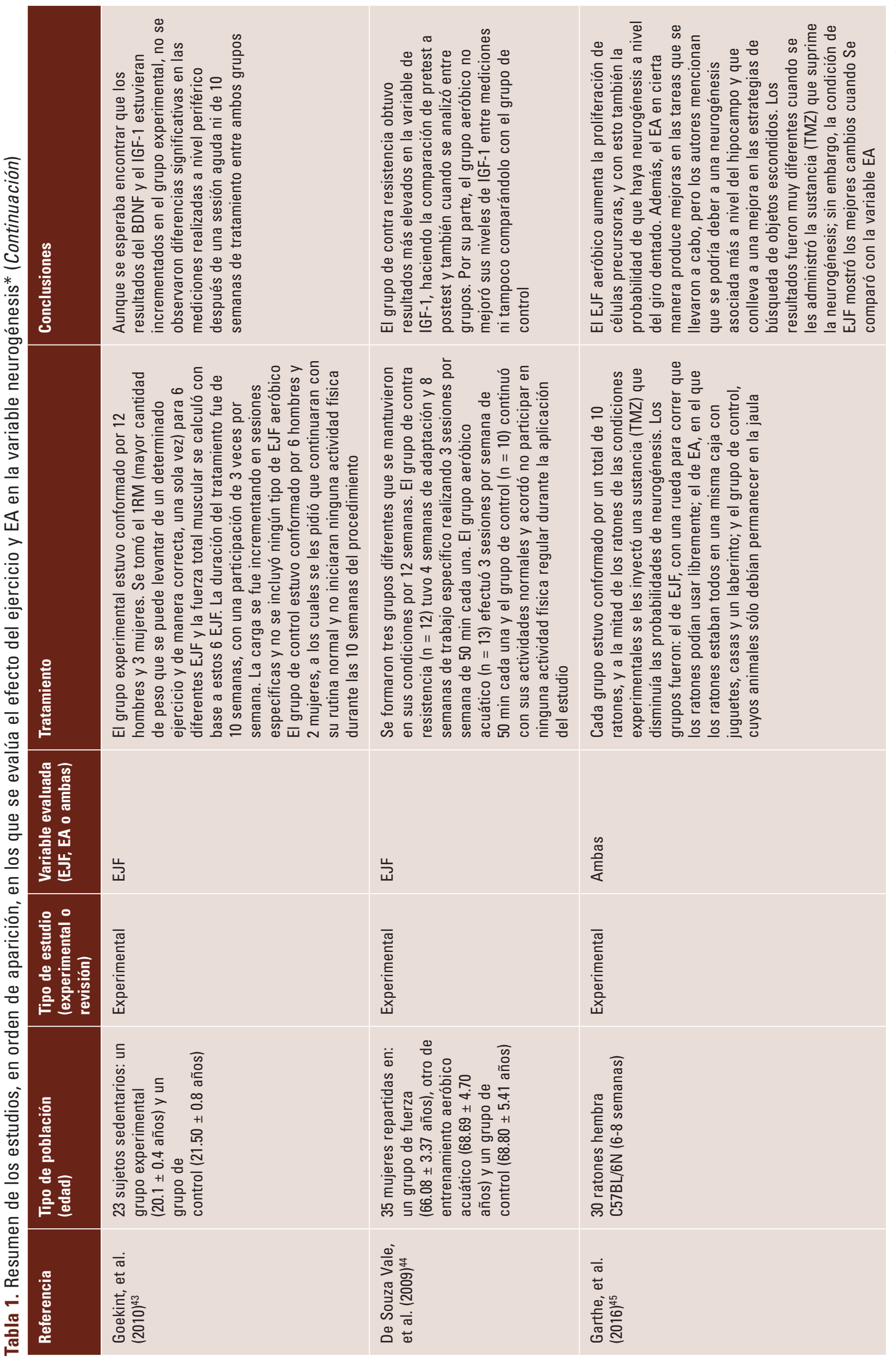




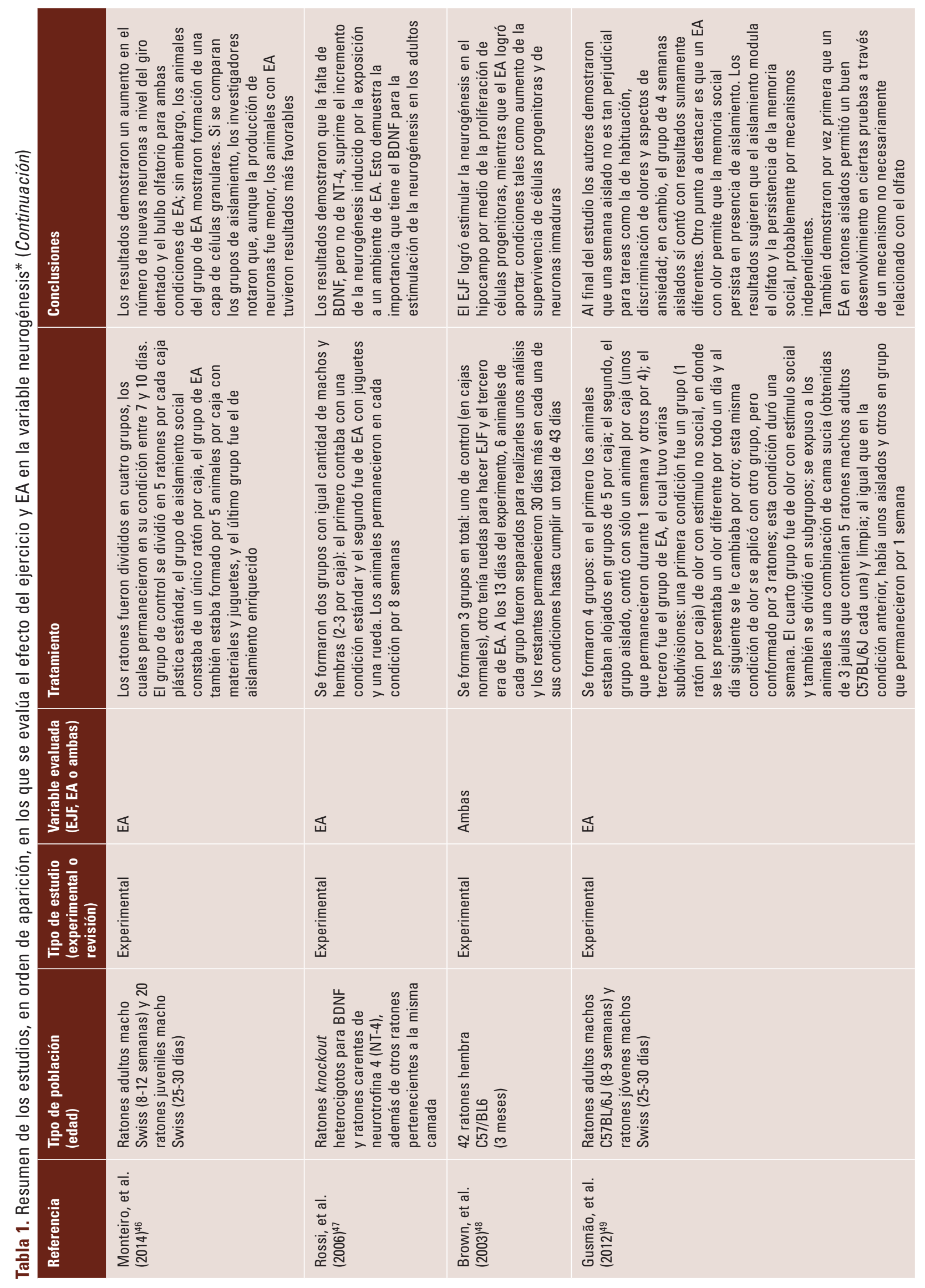




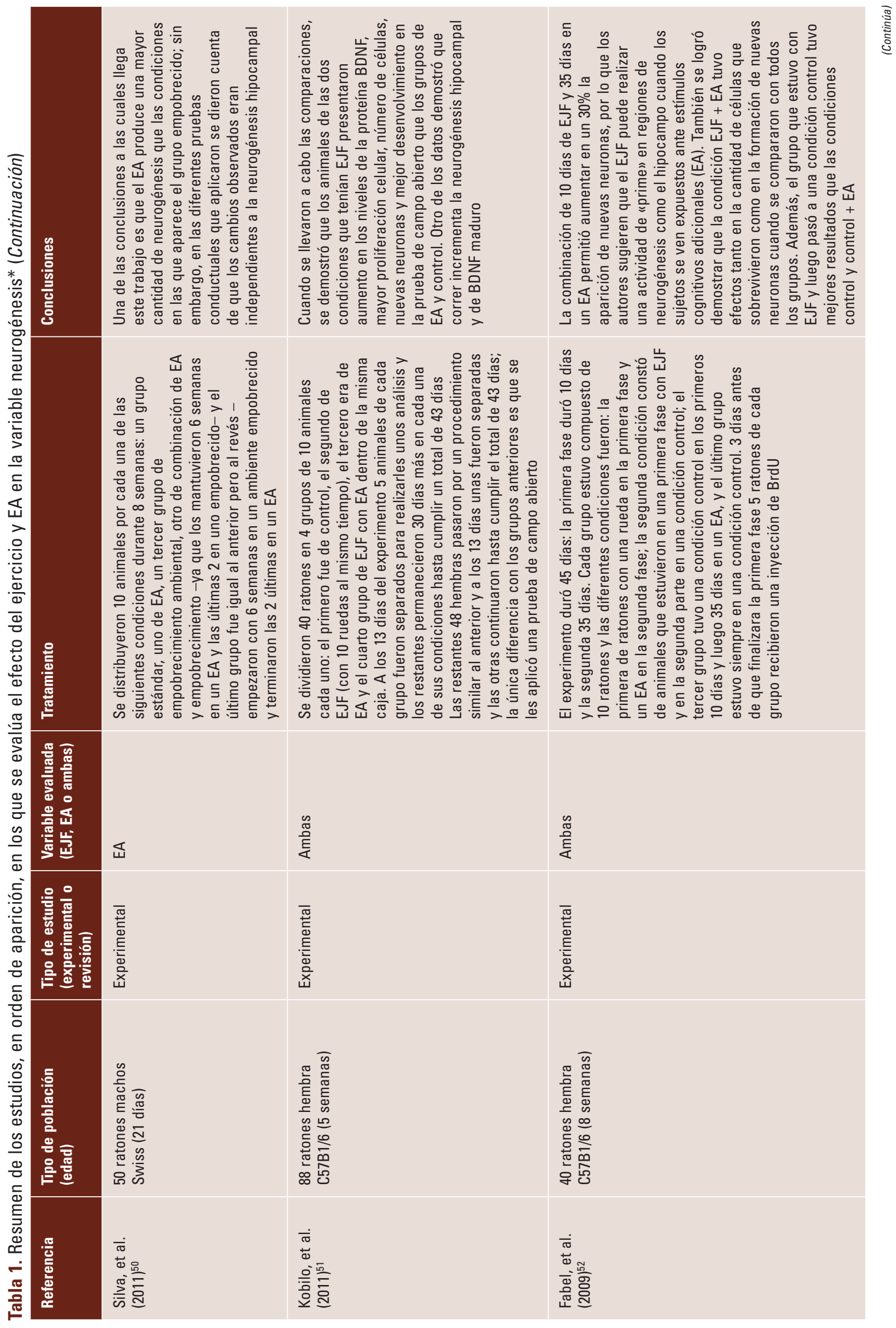




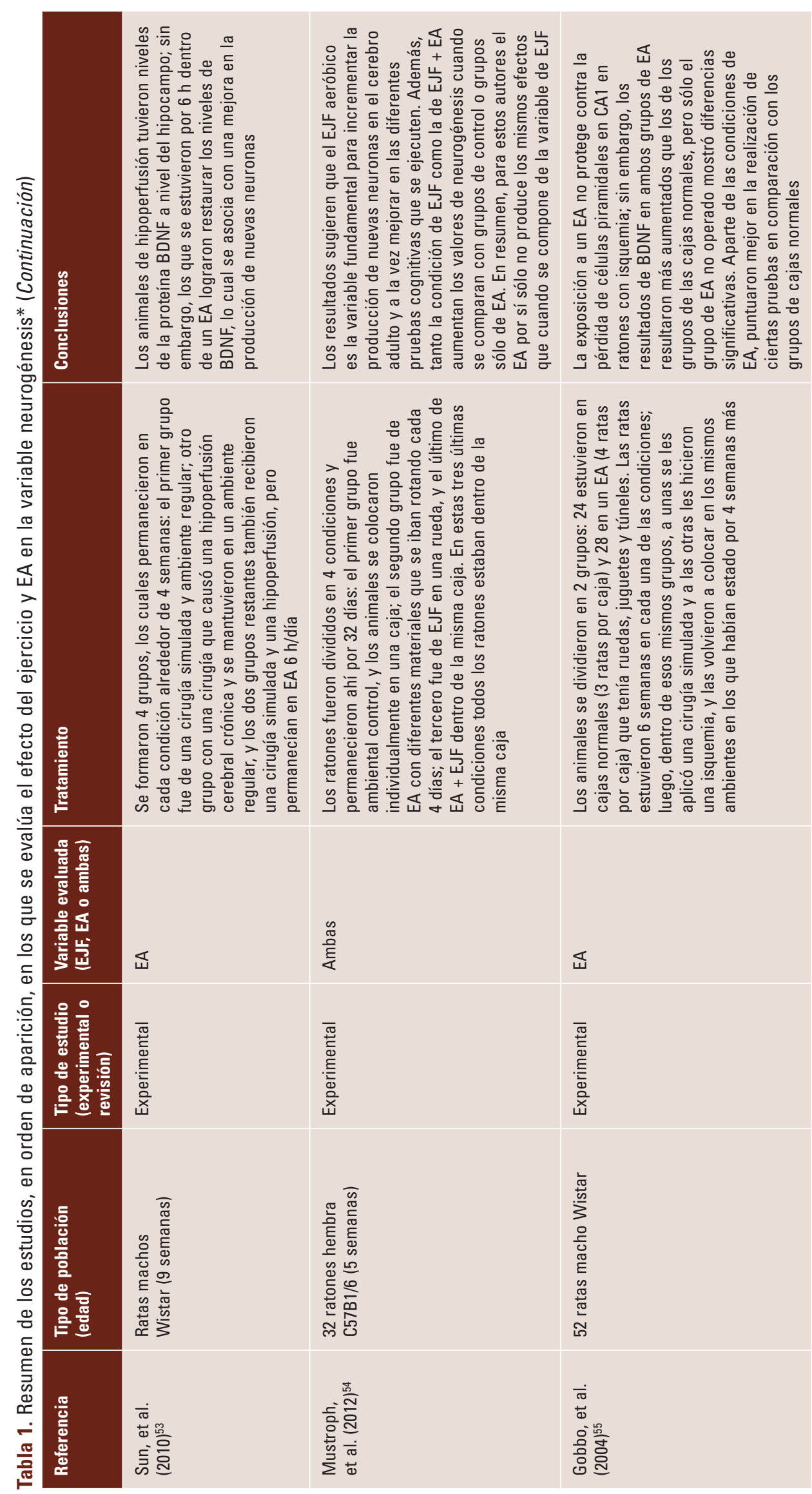




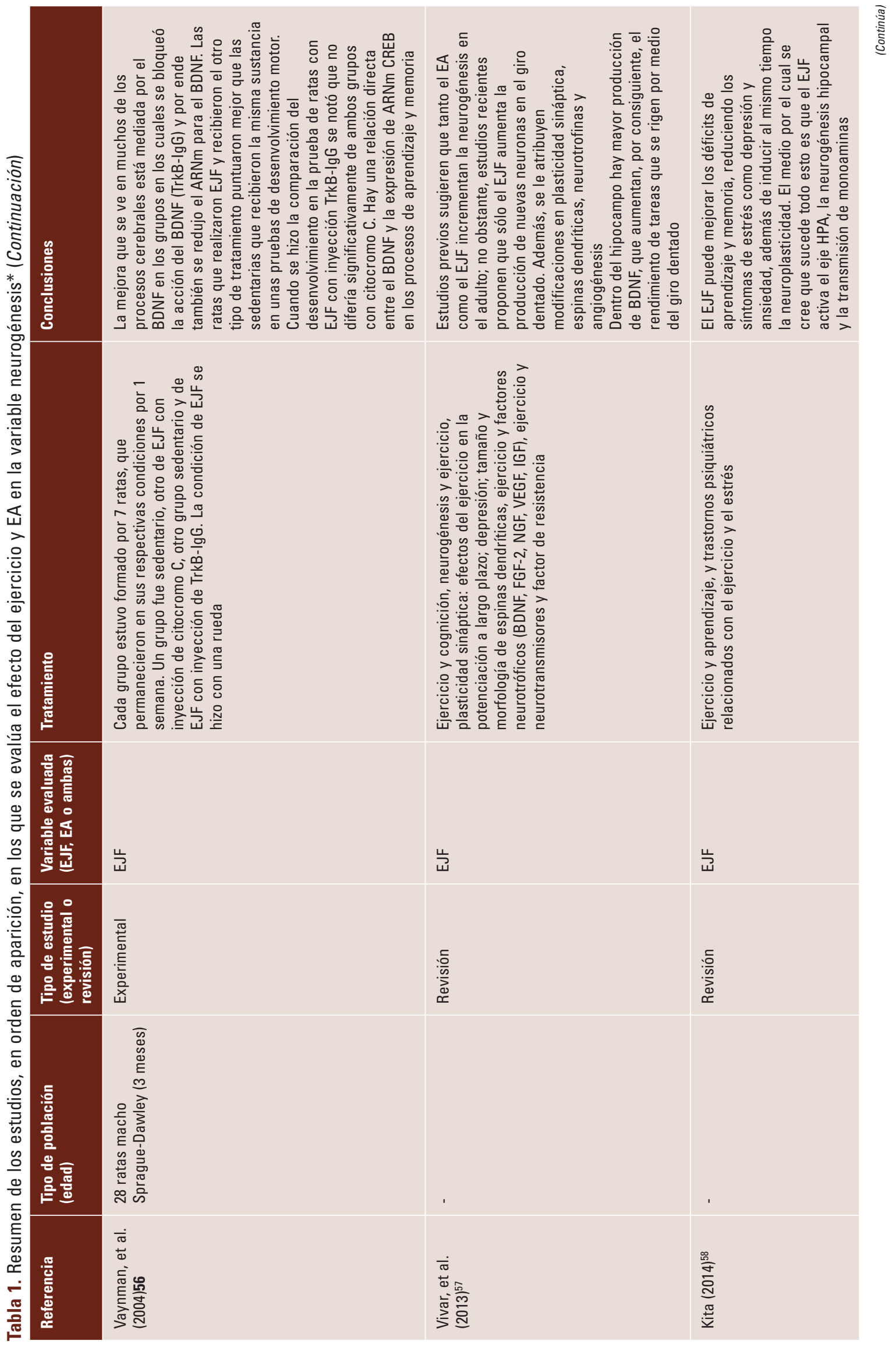




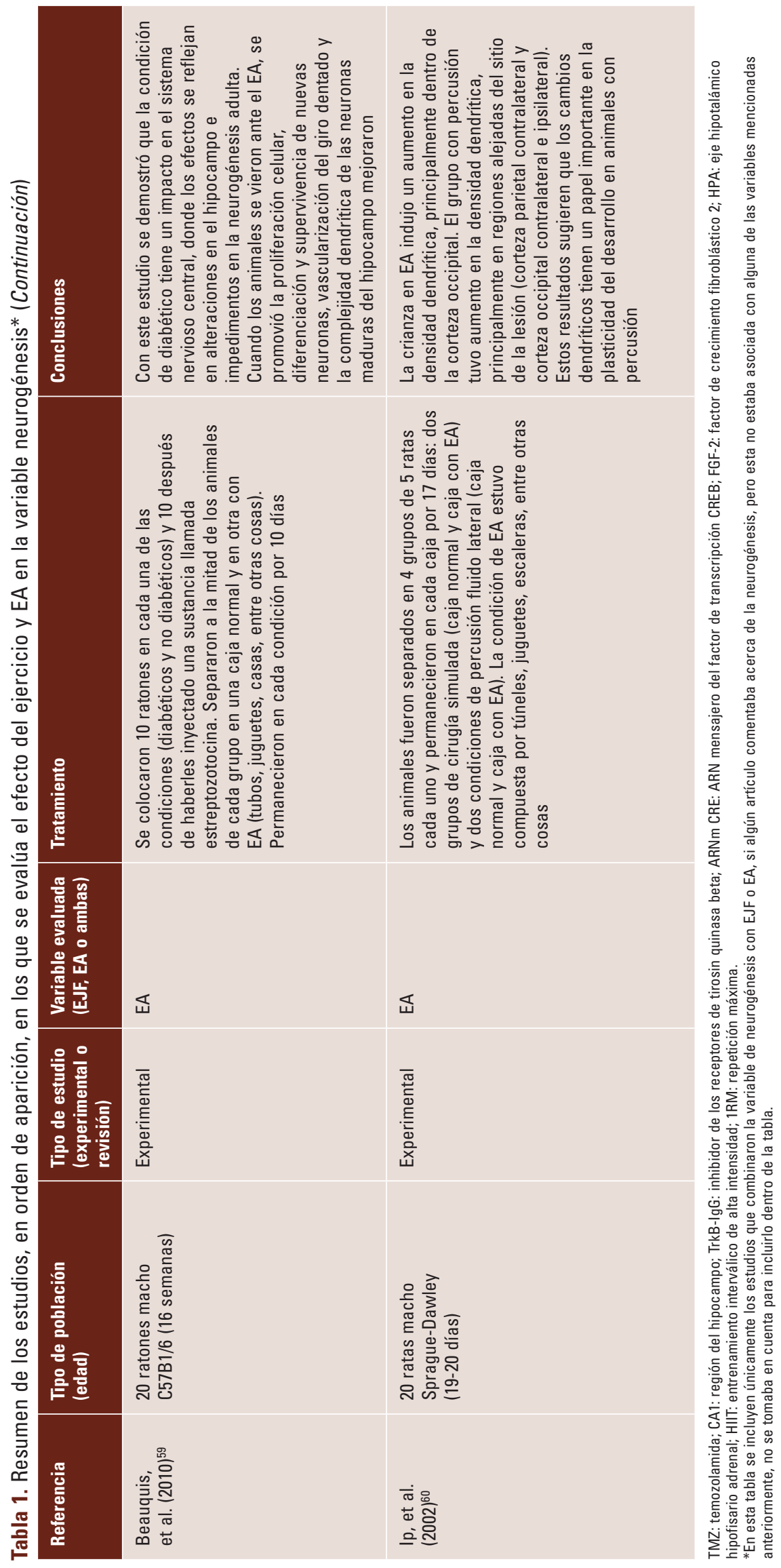


hace una combinación de EJF y EA, ya que juntos es cuando se notan más cambios.

En materia de neurogénesis, EJF, EA y estudios en humanos falta mucho por probar, aunque los modelos animales han demostrado que se producen cambios. Pero ahora hay que intentar observar esas modificaciones en las personas, y para ello hay que aplicar metodologías en las que diversas variables de EJF (duración, intensidad, frecuencia o tipo de actividad) sean analizadas con diferentes poblaciones. También es necesario incluir el EA, pues también se ha visto que tiene efectos y que hay dos variables a tomar en consideración, las cuales pueden ser medidas individual o conjuntamente, y aún más importante es notar qué se genera a nivel de la persona cuando las dos variables (EJF y EA) entran en juego.

\section{Conclusiones}

La neurogénesis es un proceso que se ha estudiado, y se estudia, con diferentes enfoques. Uno de ellos se refiere a cómo puede modularse $o$ favorecerse, y en este contexto los efectos del EJF y el EA han sido posiblemente los más investigados. De lo discutido durante el presente trabajo es fundamental mencionar que ambas variables, es decir, el EJF y el EA, favorecen la neurogénesis; sin embargo, la combinación de ambas condiciones es la que provoca el efecto más significativo. Los resultados reportados en la literatura médica no siempre concuerdan, principalmente en la magnitud del efecto, pero es posible que ello se deba a la gran cantidad de metodologías diferentes, a los tipos y duración del EJF utilizado y a la variante de EA implementada (físico, social, físico/social).

Con respecto a la relevancia que el EJF tiene en la modulación de la neurogénesis, aún quedan muchos vacíos por cubrir que posiblemente solucionarían en parte las inconsistencias encontradas en la bibliografía: hay que sistematizar la duración de las diferentes sesiones de EJF, definir mejor los circuitos de entrenamiento donde se combinen varios EJF y se tomen en cuenta variables como la hidratación, la privación del sueño, la nutrición y la ingesta de suplementos alimenticios que, de cierta forma, podrían influir en la magnitud de la neurogénesis en la edad adulta.

\section{Declaración de conflictos de interés}

No existen potenciales conflictos de interés para el autor en este informe científico.

\section{Fuentes de financiamiento}

El autor no ha declarado fuente alguna de financiamiento para este informe científico.

\section{Bibliografía}

1. Álvarez C, Olivo J, Robinson O, Quintero J, Carrasco V, Ramírez R, et al. Efectos de una sesión de ejercicio aeróbico en la presión arterial de niños, adolescentes y adultos sanos. Rev Med Chile. 2013;141(2):1363-70.

2. Olmedialla A, Ortega E, Candel N. Ansiedad, depresión y práctica de ejercicio físico en estudiantes universitarias. Apunts Med Esport. 2010; 45(167): 175-80.

3. Penedo F, Dahn J. Exercise and well-being: a review of mental and physical health benefits associated with physical activity. Curr Opin Psychiatry. 2005;18(2):189-93.

4. Rincón M. Efecto del ejercicio en la variabilidad de la frecuencia cardíaca. Revista Colombiana de Medicina Física y Rehabilitación. 2010;20(1):24-32. Disponible en: http://www.revistacmfr.org/index.php/rcmfr/article/view/29/26

5. Szabo A. Acute psychological benefits of exercise: reconsideration of the placebo effect. J Ment Health. 2013;22(5):449-55.

6. Fiuza C, Garatachea N, Berger N, Lucia A. Exercise is the real polypill. Physiology (Bethesda). 2013;28(5):330-58.

7. Déry N, Goldstein A, Becker S. A role for adult hippocampal neurogenesis at multiple time scales: A study of recent and remote memory in humans. Behav Neurosci. 2015;129(4):435-49.

8. Ming GL, Song H. Adult neurogenesis in the mammalian central nervous system. Annu Rev Neurosci. 2005;28:223-50.

9. Ming GL, Song $\mathrm{H}$. Adult neurogenesis in the mammalian brain: significant answers and significant questions. Neuron. 2011;70(4):687-702.

10. Rosenzweig M, Bennett $E$, Hebert M, Morimoto H. Social grouping cannot account for cerebral effects of enriched environments. Brain Res. 1978;153(3):563-76.

11. Van Praag H, Kempermann G, Gage F. Neural consequences of environmental enrichment. Nat Rev Neurosci. 2000;1(3):191-8.

12. Welch B, Brown D, Welch A, Lin D. Isolation, restrictive confinement or crowding of rats for one year. I. Weight, nucleic acids and protein of brain regions. Brain Res. 1974;75(1):71-84.

13. Gould E, Tanapat $P$, Hastings N, Shors T. Neurogenesis in adulthood: a possible role in learning. Trends Cogn Sci. 1999;3(5):186-92.

14. Greenough W, Cohen N, Juraska J. New neurons in old brains: learning to survive? Nat Neurosci. 1999;2(3):203.

15. Spalding K, Bergmann O, Alkass K, Bernard S, Salehpour M, Huttner H et al. Dynamics of hippocampal neurogenesis in adult humans. Cell. 2013;153(6):1219-27.

16. Eriksson P, Perfilieva E, Björk T, Alborn A, Nordborg C, Peterson D, et al Neurogenesis in the adult human hippocampus. Nature Med. 1998; 4(11):1313-7.

17. Zhao C, Deng W, Gage F. Mechanisms and functional implications of adult neurogenesis. Cell. 2008;132(4):645-60.

18. Benito J, Simón C, eds. Educar para sanar: ciencia y conciencia del nuevo paradigma educativo. 1. ${ }^{a}$ ed. EE.UU.: Bubok Publishing; 2015. Disponible en: https://books.google.co.cr/books?id=7XrYCwAAQBAJ\&p$\mathrm{g}=\mathrm{PT} 17 \& \mathrm{dq}=$ proceso+de+neuroplasticidad\&hl=es\&sa=X\&redir_esc=y\#$\mathrm{v}=$ onepage $\& \mathrm{q}=$ proceso $\% 20 \mathrm{de} \% 20$ neuroplasticidad\& $\mathrm{f}=$ false

19. Rosenzweig M, Bennett $E$. Psychobiology of plasticity: effects of training and experience on brain and behavior. Behav Brain Res. 1996;78(1):57-65.

20. Gage F. Neurogenesis in the adult brain. J Neurosci. 2002;22(3):612-3. Disponible en: http://www.jneurosci.org/content/jneuro/22/3/612.full.pdf

21. Gage F, Van Praag H. Sección 1: Neurogenesis in adult brain. En: Davis K, Charney D, Coyle J, Nemeroff C, editores. Neuropsychopharmacology.

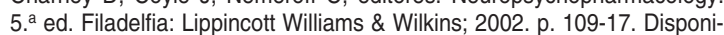
ble en: http://www.acnp.org/publications/neuro5thgeneration.aspx

22. Sirerol M, García J. Células madre y neurogénesis en el cerebro adulto. En: Lazo P, Sánchez I, editores. Medicina regenerativa y células madre. 1. ${ }^{\text {a }}$ ed. Madrid: Arbor; 2010. p. 79-104. Disponible en: https://books. google.co.cr/books?id=XDOZWsASVBkC\&printsec=frontcover\&hl=es\#$\mathrm{v}=$ onepage \&q\&f=false

23. Ramírez G, Benítez G, Kempermann G. Formación de neuronas nuevas en el hipocampo adulto: neurogénesis. Salud Mental. 2007;3(3):12-9.

24. Altman J. Are new neurons formed in the brains of adult mammals? Sciences. 1962;135(3509):1127-8.

25. Altman J, Das G. Post-natal origin of microneurones in the rat brain. Nature. 1965;207(5000):953-6.

26. Sierra A, Encinas J, Maletic M. Adult human neurogenesis: from microscopy to magnetic resonance imaging. Front Neurosci. 2011:5(47):1-18.

27. Van Praag H, Kempermann G, Gage F. Running increases cell proliferation and neurogenesis in the adult mouse dentate gyrus. Nat Neurosci. 1999;2(3):266-70. 


\section{B. Montero Herrera: Neurogénesis, ejercicio y enriquecimiento ambiental}

28. Gómez F, Ying Z, Roy R, Molteni R, Edgerton V. Voluntary exercise induce a BDNF mediated mechanism that promotes neuroplasticity. J Neurophysiol. 2002;88(5):2187-95.

29. Nokia M, Lensu S, Ahtiainen J, Johansson P, Koch L, Britton S, et al. Physical exercise increases adult hippocampal neurogenesis in male rats provided it is aerobic and sustained. J Physiol. 2016;7(594):1855-73.

30. Olson A, Eadie B, Ernst C, Christie B. Environmental enrichment and voluntary exercise massively increase neurogenesis in the adult hippocampus via dissociable pathways. Hippocampus. 2006;16(3):250-60.

31. Davenport M, Hogan D, Eskes G, Longman R, Poulin M. Cerebrovascular reserve: the link between fitness and cognitive function? Exerc Sport Sci Rev. 2012;40(3):153-8.

32. Swain R, Harris A, Wiener E, Dutka M, Morris H, Theien B, et al. Prolonged exercise induces angiogenesis and increases cerebral blood volume in primary motor cortex of the rat. Neuroscience. 2003;117(4): 1037-46.

33. Vaynman S, Ying Z, Gómez-Pinilla F. Hippocampal BDNF mediates the efficacy of exercise on synaptic plasticity and cognition. Eur J Neurosci. 2004;20(10):2580-90.

34. Vissing J, Andersen M, Diemer N. Exercise-induced changes in local cerebral glucose utilization in the rat. $\mathrm{J}$ Cereb Blood Flow Metab. 1996;16(4):729-36

35. Acevedo C, Ávila J, Cárdenas L. Efectos del ejercicio y la actividad motora sobre la estructura y función cerebral. Revista Mexicana de Neurociencia. 2014;15(1):36-53. Disponible en: http://www.medigraphic. com/pdfs/revmexneu/rmn-2014/rmn141f.pdf

36. Hötting K, Röder, B. Beneficial effects of physical exercise on neuroplasticity and cognition. Neurosci Biobehav Rev. 2013:37(9 Pt B):2243-57.

37. Lee T, Wong M, Lau B, Lee J, Yau S, So K. Aerobic exercise interacts with neurotrophic factors to predict cognitive functioning in adolescents. Psychoneuroendocrinology. 2014;39:214-24.

38. Ferreira A, Real C, Rodrígues A, Alves A, Britto L. Short-term, moderate exercise is capable of inducing structural, BDNF-independent hippocampal plasticity. Brain Res. 2011;1425:111-22.

39. Cassilhas R, Lee K, Fernándes J, Oliveira M, Tufik S, Meeusen R, et al Spatial memory is improved by aerobic and resistance exercise through divergent molecular mechanisms. Neuroscience. 2012;202:309-17.

40. Johnson R, Rhodes J, Jeffreya S, Garland T, Mitchella G. Hippocampal brain-derived neurotrophic factor but not neurotrophin-3 increases more in mice selected for increased voluntary wheel running. Neuroscience. 2003;121(1):1-7.

41. Arikawa A, Kurzer M, Thomas W, Schmitz K. No effect of exercise on insulin-like growth factor-I, insulin, and glucose in young women participating in a 16-week randomized controlled trial. Cancer Epidemiol Biomarkers Prev. 2010;19(11):2987-90.

42. Correia $P$, Pansani A, Machado F, Andrade M, Silva A, Scorza F, et al. Acute strength exercise and the involvement of small or large muscle mass on plasma brain-derived neurotrophic factor levels. Clinics (São Paulo, Brazil). 2010;65(11):1123-6.

43. Goekint M, De Pauw K, Roelands B, Njemini R, Bautmans I, Mets T, et al. Strength training does not influence serum brain-derived neurotrophic factor. Eur J Appl Physiol. 2010;110(2):285-93.

44. De Souza Vale $R$, de Oliveira R, Pernambuco $C$, de Meneses $Y$, Novaes J, de Andrade A. Effects of muscle strength and aerobic training on basal serum levels of IGF-1 and cortisol in elderly women. Arch Gerontol Geriatr. 2009;49(3):343-7.

45. Garthe A, Roeder I, Kempermann G. Mice in an enriched environment learn more flexibly because of adult hippocampal neurogenesis. Hippocampus. 2016;26(2):261-71.

46. Monteiro B, Moreira F, Massensini A, Moraes M, Pereira G. Enriched environment increases neurogenesis and improves social memory persistence in socially isolated adult mice. Hippocampus. 2014;24(2):239-48.

47. Rossi C, Angelucci A, Costantin L, Braschi C, Mazzantini M, Babbini F, et al. Brain-derived neurotrophic factor (BDNF) is required for the enhancement of hippocampal neurogenesis following environmental enrichment. Eur J Neurosci. 2006;24(7):1850-6.

48. Brown J, Cooper C, Kempermann G, Van Praag H, Winkler J, Gage F, et al. Enriched environment and physical activity stimulate hippocampal but not olfactory bulb neurogenesis. Eur J Neurosci. 2003;17(10):2042-6.

49. Gusmão I, Monteiro B, Cornélio G, Fonseca C, Moraes, M, Pereira G. Odor-enriched environment rescues long-term social memory but does not improve olfaction in social isolated adult mice. Behavioural Brain Research. 2012;228(2):440-6

50. Silva C, Duarte F, Lima T, de Oliveira C. Effects of social isolation and enriched environment on behavior of adult Swiss mice do not require hippocampal neurogenesis. Behav Brain Res. 2011;225(1):85-90.

51. Kobilo T, Liu Q, Gandhi K, Mughal M, Shaham Y, van Praag H. Running is the neurogenic and neurotrophic stimulus in environmental enrichment. Learn Mem. 2011;18(9):605-9.

52. Schloesser R, Lehmann M, Martinowich K, Manji H, Herkenham M. Environmental enrichment requires adult neurogenesis to facilitate the recovery from psychosocial stress. Mol Psychiatry. 2010;15(12):1152-63.

53. Sun $\mathrm{H}$, Zhang J, Zhang L, Liu $\mathrm{H}$, Zhu $\mathrm{H}$, Yang $\mathrm{Y}$. Environmental enrichment influences BDNF and NR1 levels in the hippocampus and restores cognitive impairment in chronic cerebral hypoperfused rats. Curr Neurovasc Res. 2010;7(4):268-80.

54. Mustroph M, Chen S, Desai S, Cay E, Deyoung E, Rhodes J. Aerobic exercise is the critical variable in an enriched environment that increases hippocampal neurogenesis and water maze learning in male C57bl/6j mice. Neuroscience. 2012;219:62-71.

55. Gobbo O, O'Mara S. Impact of enriched-environment housing on brain-derived neurotrophic factor and on cognitive performance after a transient global ischemia. Behav Brain Res. 2004;152(2):231-41.

56. Vaynman S, Ying Z, Gómez-Pinilla F. Exercise induces BDNF and synapsin I to specific hippocampal subfields. J Neurosci Res. 2004; 76(3):356-62.

57. Vivar $\mathrm{C}$, Potter $\mathrm{M}$, van Praag $\mathrm{H}$. All about running: synaptic plasticity, growth factors and adult hippocampal neurogenesis. Curr Top Behav Neurosci. 2013;15:189-210.

58. Kita I. Physical exercise can induce brain plasticity and regulate mental function. Advances In Exercise \& Sports Physiology. 2014;20(1):1-7.

59. Beauquis J, Roig P, de Nicola A, Saravia F. Short-term environmental enrichment enhances adult neurogenesis, vascular network and dendritic complexity in the hippocampus of type 1 diabetic mice. PloS One. 2010; 5(11):e13993.

60. Ip E, Giza C, Griesbach G, Hovda D. Effects of enriched environment and fluid percussion injury on dendritic arborization within the cerebral cortex of the developing rat. J Neurotrauma. 2002;19(5):573-85. 